\title{
A tribute to Guosen Yan
}

\author{
Hua Guo $\cdot$ Daiqian Xie $\cdot$ Weitao Yang
}

Published online: 15 October 2014

(C) Springer-Verlag Berlin Heidelberg 2014

Professor Guosen Yan was born in 1930 in Nanchong, Sichuan Province, China. He graduated in 1951 from Chongqing University, majoring in Chemistry, and upon graduation joined the faculty in the same university. In 1953, he was transferred to Department of Chemistry in Sichuan University, rising through the ranks and became Associate Professor in 1962 and Full Professor in 1979. He served as the President of Sichuan University from 1984 to 1989. His leadership helped to secure Sichuan University as one of the top comprehensive universities in China. Professor Yan has also served as a director and executive director of the Chinese Chemical Society and in many other important posts in China, helping to lay a solid foundation for the ongoing expansion of the Chinese research enterprise.

Published as part of the special collection of articles celebrating the 85th birthday of Professor Guosen Yan.

H. Guo $(\bowtie)$

Department of Chemistry and Chemical Biology, University

of New Mexico, Albuquerque, NM 87131, USA

e-mail: hguo@unm.edu

D. Xie

Institute of Theoretical and Computational Chemistry, Key Laboratory of Mesoscopic Chemistry, School of Chemistry and Chemical Engineering, Nanjing University, Nanjing 210093, China

W. Yang

Department of Chemistry, Duke University, Durham, NC 27708, USA

\section{W. Yang}

Key Laboratory of Theoretical Chemistry of Environment, Ministry of Education, School of Chemistry and Environment, South China Normal University, Guangzhou 510006, China
Professor Yan is a pioneer in Theoretical Chemistry in China. He was one of eight original students of Professor Au-chin Tang, a Columbia-trained theoretical chemist, widely considered as the founding father of Theoretical Chemistry in China. Professor Yan's research interests spanned several areas, including crystal field theory, graph theory, quantum chemistry, and molecular spectroscopy. His major scientific contributions include (1) analysis of the symmetry and orthogonalization of the rotational group-point group coupling coefficients in ligand field theory, (2) graph theory for determinant expansions, (3) a vibrational self-consistent field/configuration interaction theory, and (4) molecular spectroscopy and potential energy surfaces. He has written three books and close to 200 journal articles.

Professor Yan has also been a great educator. His lectures were legendary. Whether it is quantum mechanics or group theory, he presented the materials in his characteristically meticulous fashion and filled the board with perfectly written derivations and equations, never miswriting or erasing anything. He has educated more than 20 graduate students (see list), many of whom have become accomplished scientists in China. His deep understanding of quantum mechanics and group theory greatly influenced his students.

In addition to being an accomplished scientist, Professor Yan also epitomizes a classical Confucius scholar. He read widely Chinese history, poetry, literature, and philosophy. Professor Yan is also an accomplished Chinese calligrapher. He has not only influenced many researchers through his scientific insights, but also helped many of us to shape our views of science, philosophy, and life.

On this occasion of Professor Yan's 85th birthday, we celebrate his accomplishments in science and education in this Special Collection. The breadth and depth of the work reported here are testaments of the vitality of theoretical 
and computational chemistry in China, the fruits of the seeds sown by Professor Yan and his contemporaries.

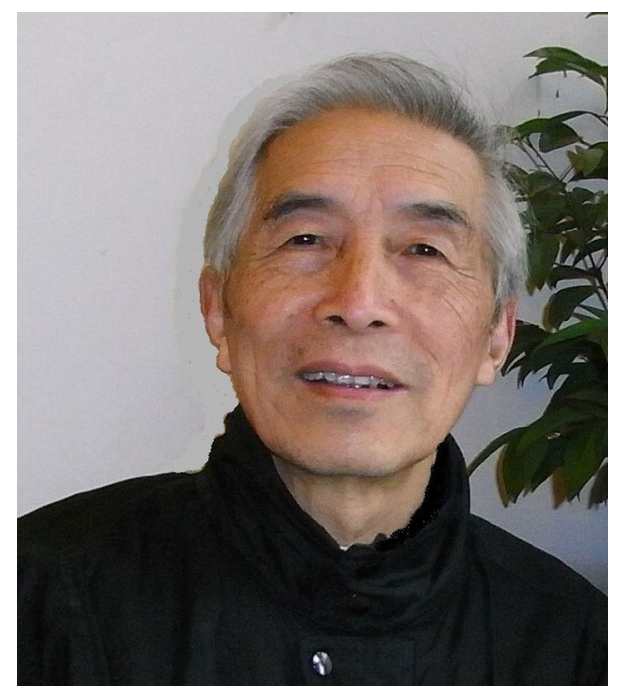

List of students of Professor Guosen Yan

\begin{tabular}{|c|c|c|c|c|}
\hline & Year & First name & Last name & 中文姓名 \\
\hline \multirow[t]{12}{*}{ M.S. } & 1978 & Yaoming & Xie & 谢尧明 \\
\hline & \multirow[t]{2}{*}{1981} & Kaiqin & Lao & 劳开勤 \\
\hline & & Huai & Sun & 孙 淮 \\
\hline & \multirow[t]{3}{*}{1982} & Daping & $\mathrm{Cao}$ & 曹大平 \\
\hline & & Hua & Guo & 郭 华 \\
\hline & & Zhi & $\mathrm{He}$ & 何 知 \\
\hline & \multirow[t]{2}{*}{1983} & Gaoqing & Yuan & 袁高清 \\
\hline & & Chongde & Zheng & 郑重德 \\
\hline & \multirow[t]{4}{*}{1984} & Ruifeng & Liu & 刘瑞峰 \\
\hline & & Kehe & $\mathrm{Su}$ & 苏克和 \\
\hline & & Xingquan & Xiang & 向兴全 \\
\hline & & Xuefeng & Zhou & 周学峰 \\
\hline \multirow[t]{13}{*}{ Ph.D. } & 1987 & $\mathrm{Jie}$ & Yang & 杨 捷 \\
\hline & 1988 & Changyuan & Tao & 陶长元 \\
\hline & 1989 & Guoming & Liang & 梁国明 \\
\hline & \multirow[t]{2}{*}{1990} & Zexing & Cao & 曹泽星 \\
\hline & & Huabei & Zhang & 张华北 \\
\hline & 1991 & Junkai & Xie & 谢军楷 \\
\hline & \multirow[t]{2}{*}{1992} & Xiangyuan & $\mathrm{Li}$ & 李象远 \\
\hline & & Jiande & $\mathrm{Gu}$ & 顾建德 \\
\hline & 1993 & Hui & Xian & 先 晖 \\
\hline & \multirow[t]{2}{*}{1994} & Ying & Xue & 薛 英 \\
\hline & & Minghui & Yang & 杨明晖 \\
\hline & 1995 & Yi & Ren & 任 译 \\
\hline & 1996 & Xuejun & $\mathrm{Xu}$ & 徐学军 \\
\hline
\end{tabular}

\section{Publications of Professor Guosen Yan}

1. G. S. Yan and M. X. Карапетъянц, J. Sichuan U. 4, 51-54 (1959).

2. М. X. Карапетъянц and G. S. Yan, J. Sichuan U. 6, 65-70 (1959).

3. М. X. Карапетъянц and G. S. Yan, J. Sichuan U. 6, 71-76 (1959).

4. М. X. Карапетьянц and G. S. Yan, Zurnal. fiz. Chem. (russ) 34, 1647 (1960).

5. М. Х. Карапетьянц and G. S. Yan, J. High. Edu. (russ) 4, 99 (1960).

6. G. S. Yan and M. X. Карапетьянц, J. Sichuan U. 1, 103-106 (1962). Zurnal. fiz. Chem. (russ) 34, 1647 (1960).

7. М. X. Карапетьянц and G. S. Yan, J. High. Edu. (russ) 4, 480 (1961).

8. G. S. Yan and M. X. Карапетъянц, J. Sichuan U. 1, 93-103 (1962). Zurnal. fiz. Chem. (russ) 37, 2041 (1963).

9. Academic discussion on structural chemistry, Acta Sci. Nat. U. Jilin. 3, 79-100 (1964).

10. Academic discussion on structural chemistry, Acta Sci. Nat. U. Jilin. 1, 59-70 (1965).

11. A. Q. Tang and G. S. Yan, Theoretical Method of the Ligand Field Theory, Science Press, Beijing (1979).

12. A. Q. Tang, Y. S. Jiang, G. S. Yan and S. S. Dai, Graph Theoretical Molecular Orbitals, Science Press, Beijing (1986).

13. G. S. Yan, Int. J. Quantum. Chem. 14, 549-550 (1980).

14. G. S. Yan, Z. M. Sun, S. X. Xiao and X. F. Zhou, J. Atom. Mol. Phys. 2, 68-83 (1985).

15. H. Sun, A. M. Tian and G. S. Yan, Int. J. Quantum Chem. 29, 1303-1324 (1986).

16. W. G. Sun, Y. M. Xie, A. M. Tian and G. S. Yan, Chem. J. Chinese U. 7, 448-452 (1986).

17. G. S. Yan, Z. M. Sun, S. X. Xiao and X. F. Zhou, Chem. J. Chinese U. 7, 351-356 (1986).

18. G. S. Yan, Z. M. Sun, S. X. Xiao, X. F. Zhou and Y. Xue, Acta Phys-Chim Sin. 2, 505-511 (1986).

19. K. Q. Lao, A. M. Tian and G. S. Yan, J. Atom. Mol. Phys. 3, 189-196 (1986).

20. C. D. Zheng, G. S. Yan, Z. M. Sun, A. M. Tian and S. X. Xiao, J. Atom. Mol. Phys. 4, 501-506 (1987).

21. H. Guo, A. M. Tian and G. S. Yan, J. Mol. Sci. (Theochem) 149, 105-109 (1988).

22. A. M. Tian, R. F. Liu, Z. M. Sun and G. S. Yan, Chem. J. Chinese U. 8, 836-837 (1988). 
23. G. S. Yan, Z. M. Sun, S. X. Xiao and Y. Xue, Chem. J. Chinese U. 9, 700-705 (1988).

24. G. Q. Yuan, A. M. Tian, Z. H. Tang and G. S. Yan, $J$. Atom. Mol. Phys. 5, 803-810 (1988).

25. A. M. Tian, R. F. Liu, Z. M. Sun, X. F. Zhou and G. S. Yan, Int. J. Quantum. Chem. 36, 765-771 (1989).

26. C. D. Zheng, G. S. Yan, Z. M. Sun and S. X. Xiao, Acta Chim. Sinica 47, 603-608 (1989).

27. A. M. Tian, G. Q. Yuan, Z. H. Tang and G. S. Yan, Chem. J. Chinese U. 10, 826-830 (1989).

28. Z. M. Sun, Z. X. Cao and G. S. Yan, Chinese J. Chem. Phys. 3, 350-356 (1990).

29. S. X. Xiao, J. Ji, T. L. Chen, T. C. Cai and G. S. Yan, J. Mol. Struct. (Theochem) 204, 33-40 (1990).

30. Z. R. Li, A. M. Tian and G. S. Yan, J. Sichuan U. (Nat. Sci. Edit.) 28, 401-402 (1991).

31. Z. M. Sun, Z. X. Cao and G. S. Yan, J. Atom. Mol. Phys. 2, 1859-1864 (1991).

32. M. Tian, G. M. Liang and G. S. Yan, Quantum Chemistry Computational Methods of Chemical Bonds, Sichuan University Press, Chengdu (1992).

33. Z. X. Cao, Z. M. Sun and G. S. Yan, J. Sichuan U. (Nat. Sci. Edit.) 29, 246-253 (1992).

34. T. L. Chen, J. Ji, S. X. Xiao, T. Cai and G. S. Yan, Int. J. Quantum. Chem. 44, 1015-1025 (1992).

35. G. S. Yan, Z. X. Cao, G. Liang and A. M. Tian, J. Mol. Struct. (Theochem) 282, 283-286 (1993).

36. A. M. Tian, Z. X. Cao, J. L. Zhang and G. S. Yan, J. Mol. Struct. (Theochem) 285, 293-296 (1993).

37. D. Q. Xie, G. S. Yan, J. K. Xie and A. M. Tian, Chinese Chem. Lett. 4, 175-178 (1993).

38. J. Yang, G. S. Yan, A. M. Tian, A. Q. Tang and Q. S. Li, Chinese Chem. Lett. 4, 93-94 (1993).

39. Z. X. Cao, A. M. Tian and G. S. Yan, Chinese Sci. Bull. 38, 233-235 (1993).

40. Z. X. Cao, G. S. Yan and A. M. Tian, Chem. J. Chinese U. 14, 711-713 (1993).

41. G. M. Liang, A. M. Tian and G. S. Yan, Chem. J. Chinese U. 14, 1705-1709 (1993).

42. Z. M. Sun, J. S. Li and G. S. Yan, Chem. J. Chinese U. 14, 1560-1564 (1993).

43. Z. X. Cao, G. M. Liang, A. M. Tian, G. S. Yan, A. Q. Tang and S. Q. Li, Acta Phys-Chim Sin. 9, 770775 (1993).

44. G. M. Liang, J. D. Gu, A. M. Tian and G. S. Yan, J. Sichuan U. (Nat. Sci. Edit.) 30, 370-374 (1993).

45. Z. H. Tang, H. P. Zhou, G. S. Yan and A. M. Tian, J. Sichuan U. (Nat. Sci. Edit.) 30, 375-379 (1993).

46. J. Yang, A. M. Tian, G. M. Liang and G. S. Yan, J. Sichuan U. (Nat. Sci. Edit.) 30, 233-237 (1993).

47. Z. X. Cao, G. M. Liang, A. M. Tian, G. S. Yan, A. Q. Tang and S. Q. Li, Chinese J. Chem. Phys. 5, 491-498 (1993).
48. Z. X. Cao, Z. M. Sun and G. S. Yan, Chem. J. Chinese U. 13, 398-400 (1993).

49. G. S. Yan, D. Q. Xie and A. M. Tian, J. Phys. Chem. 98, 8870-8875 (1994).

50. J. Yang, G. M. Liang, A. M. Tian, J. D. Gu and G. S. Yan, Acta Chim. Sinica 52, 562-566 (1994).

51. Z. X. Cao, G. M. Liang, A. M. Tian and G. S. Yan, Acta Chim. Sinica 52, 223-227 (1994).

52. X. Y. Li, F. C. He, A. M. Tian and G. S. Yan, Chem. Onl. 12, 17-21 (1994).

53. J. Yang, A. M. Tian, G. M. Liang and G. S. Yan, Chem. J. Chinese U. 15, 249-250 (1994).

54. D. Q. Xie, G. S. Yan and A. M. Tian, Chem. J. Chinese U. 15, 1220-1221 (1994).

55. J. D. Gu, A. M. Tian and G. S. Yan, Chem. J. Chinese U. 15, 886-890 (1994).

56. J. D. Gu, A. M. Tian and G. S. Yan, Chem. J. Chinese U. 15, 1222-1224 (1994).

57. H. B. Zhang, G. M. Liang, A. M. Tian and G. S. Yan, Acta Phys-Chim Sin. 10, 360-362 (1994).

58. Z. X. Cao, A. M. Tian and G. S. Yan, Acta Phys-Chim Sin. 10, 387-390 (1994).

59. H. B. Zhang, A. M. Tian and G. S. Yan, Acta PhysChim Sin. 10, 481-483 (1994).

60. J. Yang, G. M. Liang, A. M. Tian and G. S. Yan, Acta Phys-Chim Sin. 10, 367-370 (1994).

61. J. L. Zhang, Z. X. Cao, J. D. Gu, A. M. Tian and G. S. Yan, Acta Phys-Chim Sin. 10, 396-398 (1994).

62. J. Yang, G. M. Liang, A. M. Tian and G. S. Yan, Chinese J. Chem. Phys. 7, 34-38 (1994).

63. Z. H. Tang, H. P. Zhou, Z. M. Sun, G. S. Yan and A. M. Tian, Chinese J. Chem. Phys. 7, 221-226 (1994).

64. Z. X. Cao, A. M. Tian and G. S. Yan, Chinese J. Chem. Phys. 7, 437-442 (1994).

65. Z. M. Sun, J. S. Li and G. S. Yan, Chinese J. Chem. Phys. 7, 111-117 (1994).

66. Z. H. Tang, J. S. Chen, Z. M. Sun, Y. Xue, G. S. Yan and A. M. Tian, J. Sichuan U. (Nat. Sci. Edit.) 31, 232-237 (1994).

67. Z. H. Tang, L. C. Li, Z. M. Sun and G. S. Yan, J. Sichuan U. (Nat. Sci. Edit.) 31, 364-370 (1994).

68. G. M. Liang, A. M. Tian and G. S. Yan, J. Sichuan U. (Nat. Sci. Edit.) 31, 238-245 (1994).

69. Z. H. Tang, J. S. Chen, Z. M. Sun, G. S. Yan and A. M. Tian, J. Atom. Mol. Phys. 11, 232-237 (1994).

70. C. Y. Tao, J. L. Luo, Z. R. Li and G. S. Yan, J. Atom. Mol. Phys. 11, 360-368 (1994).

71. X. Y. Li, F. C. He, A. M. Tian and G. S. Yan, J. Mol. Struct. (Theochem) 342, 181-186 (1995).

72. Z. X. Cao, J. L. Zhang, A. M. Tian and G. S. Yan, J. Mol. Struct.(Theochem) 333, 191-197 (1995).

73. L. X. Zhou, J. L. Zhang, A. M. Tian and G. S. Yan, J. Mol. Struct. (Theochem) 358, 173-177 (1995). 
74. X. Y. Li, A. M. Tian, F. C. He and G. S. Yan, Chem. Phys. Lett. 233, 227-230 (1995).

75. J. D. Gu, A. M. Tian and G. S. Yan, Chinese Sci. Bull. 40, 1972-1977 (1995).

76. G. S. Yan and D. Q. Xie, Chinese Sci. Bull. 40, 1797-1800 (1995).

77. X. Y. Li, A. M. Tian, F. C. He and G. S. Yan, Chinese Sci. Bull. 40, 1698-1702 (1995).

78. D. Q. Xie, G. S. Yan and A. M. Tian, Chinese J. Chem. 13, 510-514 (1995).

79. G. P. Li, A. M. Tian and G. S. Yan, Acta Chim. Sinica. 53, 332-335 (1995).

80. H. B. Zhang, A. M. Tian and G. S. Yan, Chinese Chem. Lett. 6, 357-360 1995).

81. J. K. Xie, D. Q. Xie, G. S. Yan and A. M. Tian, Chem. J. Chinese U. 16, 1940-1944 (1995).

82. H. B. Zhang, C. W. Hu, A. M. Tian and G. S. Yan, Chem. J. Chinese U. 16, 626-628 (1995).

83. Z. H. Tang, J. S. Chen, Z. M. Sun, A. M. Tian and G. S. Yan, Chem. J. Chinese U. 16, 761-764 (1995).

84. J. L. Zhang, J. D. Gu, A. M. Tian, G. S. Yan and Y. B. Wang, Acta Chim. Sinica 53, 665-669 (1995).

85. X. Y. Li, A. M. Tian, F. C. He and G. S. Yan, Acta Chim. Sinica 53, 933-936 (1995).

86. H. B. Zhang, A. M. Tian and G. S. Yan, Acta Chim. Sinica 53, 538-542 (1995).

87. G. M. Liang, J. D. Gu, A. M. Tian and G. S. Yan, Acta Chim. Sinica 53, 961-965 (1995).

88. G. P. Li, A. M. Tian and G. S. Yan, Acta Chim. Sinica 53, 332-335 (1995).

89. G. P. Li, H. B. Zhang, A. M. Tian and G. S. Yan, Acta Phys-Chim Sin. 11, 211-217 (1995).

90. H. B. Zhang, A. M. Tian and G. S. Yan, Acta PhysChim Sin. 11, 142-146 (1995).

91. J. D. Gu, A. M. Tian and G. S. Yan, Acta Phys-Chim Sin. 11, 719-723 (1995).

92. J. Yang, Z. H. Tang, D. Y. Wu, Z. R. Li, A. M. Tian and G. S. Yan, Acta Phys-Chim Sin. 11, 1008-1013 (1995).

93. Z. R. Li, J. Yang, M. H. Yang, A. M. Tian and G. S. Yan, J. Sichuan U. (Nat. Sci. Edit.) 32, 701-105 (1995).

94. Z. H. Tang, L. C. Li, Z. M. Sun, A. M. Tian and G. S. Yan, J. Atom. Mol. Phys. 12, 190-201 (1995).

95. Z. H. Tang, Z. P. Huang, Z. M. Sun, A. M. Tian and G. S. Yan, J. Atom. Mol. Phys. 12, 293-298 (1995).

96. Z. H. Tang, L. C. Li, Z. P. Huang, Z. M. Sun, A. M. Tian and G. S. Yan, Chinese J. Syn. Chem. 3, 235-238 (1995).

97. D. Q. Xie and G. S. Yan, Chem. Phys. Lett. 248, 409-413 (1996).

98. D. Q. Xie and G. S. Yan, Mol. Phys. 88, 1349-1355 (1996).
99. Li X, A. M. Tian, F. C. He and G. S. Yan, J. Comput. Chem. 17, 1108-1111 (1996).

100. J. D. Gu, J. L. Zhang, M. A, Tian and G. S. Yan, Acta Chim. Sinica. 54, 320-324 (1996).

101. D. Q. Xie and G. S. Yan, Sci. China (B) 39, 439-448 (1996).

102. J. D. Gu, A. M. Tian and G. S. Yan, Sci. China (B) 39, 29-36 (1996).

103. Z. H. Tang, L. C. Li, Z. M. Sun, G. S. Yan and H. P. Zhou, Sci. China (B) 39, 269-275 (1996).

104. L. X. Zhou, D. L. Zhang, A. M. Tian and G. S. Yan, Chinese Chem. Lett. 7, 59-62 (1996).

105. J. K. Xie, D. Q. Xie, A. M. Tian and G. S. Yan, Acta Chim. Sinica 54, 261-265 (1996).

106. J. K. Xie, D. Q. Xie and G. S. Yan, Acta Chim. Sinica 54, 756-763 (1996).

107. J. D. Gu, J. L. Zhang, A. M. Tian and G. S. Yan, Acta Chim. Sinica 54, 320-324 (1996).

108. J. D. Gu, A. M. Tian and G. S. Yan, Acta Chim. Sinica 54, 331-337 (1996).

109. L. X. Zhou, A. M. Tian, J. C. Li, Y. Z. Chen and G. S. Yan, Chem. J. Chinese U. 17, 117-120 (1996).

110. H. B. Zhang, J. L. Zhang, Z. R. Li, A. M. Tian and G. S. Yan, J. Sichuan U. (Nat. Sci. Edit.) 33, 307-310 (1996).

111. H. B. Zhang, A. M. Tian and G. S. Yan, J. Sichuan U. (Nat. Sci. Edit.) 33, 718-722 (1996).

112. J. Yang, D. Y. Wu, Z. H. Tang, Z. R. Li, A. M. Tian and G. S. Yan, J. Sichuan U. (Nat. Sci. Edit.) 33, 723727 (1996).

113. G. S. Yan, M. H. Yang and D. Q. Xie, Chem. Phys. Lett. 275, 494-498 (1997).

114. G. S. Yan, H. Xian and D. Q. Xie, Chem. Phys. Lett. 271, 157-162 (1997).

115. G. S. Yan, M. H. Yang and D. Q. Xie, Sci. China (B) 40, 554-560 (1997).

116. G. S. Yan, H. Xian and D. Q. Xie, Sci. China (B) 40, 342-347 (1997).

117. G. S. Yan, J. K. Xie and D. Q. Xie, Chinese Sci. Bull. 42, 43-46 (1997).

118. J. K. Xie, G. S. Yan and D. Q. Xie, Chinese Sci. Bull. 42, 268-272 (1997).

119. D. Q. Xie and G. S. Yan, Chem. J. Chinese U. 18, 1136-1140 (1997).

120. H. Xian, D. Q. Xie and G. S. Yan, Acta Phys-Chim Sin. 13, 865-867 (1997).

121. G. S. Yan, H. Xian and D. Q. Xie, Sci. China (B) 27, 228-234 (1997).

122. G. S. Yan, M. H. Yang and D. Q. Xie, Sci. China $(B)$ 27, 527-533 (1997).

123. Z. M. Sun, B. Wang and G. S. Yan, Acta Chim. Sinica 55, 9-12 (1997). 
124. G. S. Yan, J. K. Xie and D. Q. Xie, Acta Chim. Sinica 55, 1041-1046 (1997).

125. G. S. Yan, H. Xian, and D. Q. Xie, Mol. Phys. 93, 867-872 (1998).

126. G. S. Yan, M. H. Yang, and D. Q. Xie, Chem. Phys. Lett. 287, 162-168 (1998).

127. G. S. Yan, M. H. Yang, and D. Q. Xie, J. Chem. Phys. 109, 10284-10292 (1998).

128. H. Xian, D. Q. Xie, and G. S. Yan, Sci. China (B) 41, 320-324 (1998).

129. H. Xian, D. Q. Xie and G. S. Yan, Sci. China (B) 28, 243-246 (1998).

130. G. S. Yan, Y. Xue, and D. Q. Xie, Sci. China (B) 41, 91-96 (1998).

131. G. S. Yan, Y. Xue, and D. Q. Xie, Int. J. Quant. Chem. 72, 53-60 (1999).

132. Y. Xue, X. J. Xu, D. Q. Xie and G. S. Yan, J. Mol. Struc. 469, 151-165 (1999).

133. M. L. Yang, Z. M. Sun, and G. S. Yan, Sci. China (B) 42, 70-76 (1999).

134. M. L. Yang, Z. M. Sun and G. S. Yan, Sci. China (B) 29, 206-211 (1999).

135. H. Zhu, D. Q. Xie and G. S. Yan, Chem. J. Chinese U. 20, 1910-1915 (1999).

136. Y. Xue, D. Q. Xie and G. S. Yan, Chem. J. Chinese U. 20, 1102-1105 (1999).

137. M. L. Yang, Z. M. Sun and G. S. Yan, Chem. J. Chinese U. 20, 450-453 (1999).

138. X. J. Xu, Y. Xue, D. Q. Xie and G. S. Yan, Chem. J. Chinese U. 20, 941-947 (1999).

139. Y. H. Lu, H. Xian, D. Q. Xie and G. S. Yan, Chem. J. Chinese U. 20, 1291-1294 (1999).

140. M. L. Yang, Z. M. Sun, D. Q. Xie and G. S. Yan, Chinese J. Chem. Phys. 12, 717-721 (1999).

141. M. L. Yang, Z. M. Sun and G. S. Yan, Chinese J. Chem. Phys. 12, 282-287 (1999).

142. M. L. Yang, Z. M. Sun and G. S. Yan, J. Atom. Mol. Phys. 16, 413-417 (1999).

143. M. L. Yang, Z. M. Sun and G. S. Yan, Acta Phys-Chim Sin. 15, 693-697 (1999).

144. Y. Xue, D. Q. Xie and G. S. Yan, Acta Phys-Chim Sin. 15, 138-142 (1999).

145. M. L. Yang, Z. M. Sun and G. S. Yan, Acta Chim. Sinica 57, 754-759 (1999).

146. X. J. Xu, Y. Xue, D. Q. Xie and G. S. Yan, Acta Chim. Sinica 57, 680-684 (1999).

147. Y. H. Lu, D. Q. Xie, M. H and G. S. Yan, Chem. Phys. Lett. 327, 305-313 (2000).

148. Y. Xue, D. G. Xu, D. Q. Xie and G. S. Yan, Spectrochim Acta A 56, 1929-1938 (2000).

149. X. J. Xu, D. Q. Xie, Y. Xue and G. S. Yan, Spectrochim Acta A 56, 567-574 (2000).
150. M. H. Yang, D. Q. Xie and G. S. Yan, Sci. China (B) 43, 196-200 (2000).

151. Y. Xue, D. Q. Xie, and G. S. Yan, Int. J. Quant. Chem. 76, 86-699 (2000).

152. Y. H. Lu, D. Q. Xie, and G. S. Yan, Int. J. Quant. Chem. 78, 269-280 (2000).

153. Y. Xue, D. G. Xu, D. Q. Xie and G. S. Yan, Chem. J. Chinese U. 21, 1293-1298 (2000).

154. D. G. Xu, Y. H. Lu, D. Q. Xie and G. S. Yan, Chem. J. Chinese U. 21, 1884-1887 (2000).

155. H. Y. Xiao, Y. Xue, X. J. Xu, D. Q. Xie and G. S. Yan, Acta Chim. Sinica 58, 414-417 (2000).

156. Y. Xue, Y. Guo, X. J. Xu, D. Q. Xie and G. S. Yan, Acta Chim. Sinica 58, 1254-1258 (2000).

157. Y. H. Lu, Y. Z. Zhou, D. Q. Xie and G. S. Yan, Acta Chim. Sinica 58, 1516-1521 (2000).

158. M. H. Yang, D. Q. Xie and G. S. Yan, Sci. China (B) 30, 28-32 (2000).

159. J. K. Xie and G. S. Yan, Chinese Sci. Bull. 45, 2606-2610 (2000).

160. D. Q. Xie, Y. H. Lu, D. G. Xu and G. S. Yan, Chem. Phys. 270, 415-428 (2001).

161. D. Q. Xie, Y. H. Lu and G. S. Yan, Chem. Phys. Lett. 339, 14-22 (2001).

162. J. K. Xie and G. S. Yan, Chin. Sci. Bull. 46, 650-652 (2001).

163. Y. Xue, D. Q. Xie and G. S. Yan, Chem. J. Chinese U. 22, 1189-1192 (2001).

164. Y. Guo, D. Q. Xie, Y. Xue and G. S. Yan, Chem. J. Chinese U. 22, 2061-2064 (2001).

165. H. Zhu, D. Q. Xie and G. S. Yan, Chem. Phys. Lett. 351, 149-157 (2002).

166. C. Zhou, D. Q. Xie, R. Q. Chen, G. S. Yan and H. Guo, Spectrochim Acta A 58, 727-746 (2002).

167. H. Zhu, J. Li, D. Q. Xie and G. S. Yan, Chem. J. Chinese U. 23, 2137-2141 (2002).

168. Y. Guo, D. Q. Xie, Y. Xue and G. S. Yan, Acta Chim. Sinica 60, 660-663 (2002).

169. Y. Wu, K. L. Xu, Y. Xue, D. Q. Xie and G. S. Yan, Acta Chim. Sinica 60, 2092-2096 (2002).

170. H. Zhu, D. Q. Xie and G. S. Yan, J. Chem. Phys. 24, 1839-1845 (2003).

171. J. Li, D. Q. Xie and G. S. Yan, Sci. China (B) 46, 113-118 (2003).

172. H. Y. Xiao, W. Z. Lai, D. Q. Xie and G. S. Yan, Sci. China (B) 46, 425-430 (2003).

173. J. Li, H. Zhu, D. Q. Xie and G. S. Yan, Chem. J. Chinese U. 24, 686-689 (2003).

174. K. L. Xu, D. Q. Xie and G. S. Yan, Chem. J. Chinese U. 24, 2031-2034 (2003).

175. H. Zhang, Y. Xue, K. L. Xu, D. Q. Xie and G. S. Yan, Acta Chim. Sinica 61, 1926-1929 (2003). 
176. J. Li, D. Q. Xie, G. S. Yan and D. Q. Xie, Sci. China (B) 33, 21-25 (2003).

177. H. Y. Xiao, W. Z. Lai, D. Q. Xie and G. S. Yan, Sci. China (B) 33, 239-244 (2003).

178. J. X. Chen, H. Zhu, D. Q. Xie and G. S. Yan, Acta Chim. Sinica 62, 5-9 (2004).

179. K. L. Xu, Y. Wu, D. Q. Xie and G. S. Yan, Chinese Sci. Bull. 49, 535-537 (2004).

180. Y. Z. Zhou, H. Zhu, D. Q. Xie and G. S. Yan, Chem. J. Chinese U. 25, 1081-1084 (2004).

181. K. L. Xu, D. Q. Xie and G. S. Yan, Acta Chim. Sinica 62, 2003-2006 (2004).

182. Y. Xue, H. Zhang, D. Q. Xie and G. S. Yan, Chem. J. Chinese U. 26, 907-911 (2005).

183. Y. Guo, Y. Xue, D. Q. Xie and G. S. Yan, Chem. J. Chinese U. 26, 1686-1689 (2005).

184. H. Zhang, Y. Xue, D. Q. Xie and G. S. Yan, Acta Chim. Sinica 63, 791-796 (2005).

185. L. Jin, Y. Wu, Y. Xue, Y. Guo, D. Q. Xie and G. S. Yan, Acta Chim. Sinica 64, 873-878 (2006).
186. B. He, Y. Xue, Y. Guo and G. S. Yan, Acta Chim. Sinica 65, 481-488 (2007).

187. Y. Zeng, Y. Xue and G. S. Yan, J. Phys. Chem. B 112, 10659-10667 (2008).

188. D. G. Xu and G. S. Yan, Chem. J. Chinese U. 29, 2453-2456 (2008).

189. C. H. Zhang, Y. Xue, Y. Guo and G. S. Yan, Chem. J. Chinese U. 29, 2354-2359 (2008).

190. Y. Y. Sun, C. H. Zhang, Y. Xue and G. S. Yan, Acta Chim. Sinica 66, 751-756 (2008).

191. J. L. Liu, Y. Guo, Y. Xue and G. S. Yan, Chem. J. Chinese U. 30, 100-105 (2009).

192. R. Chen, H. Zhu, D. Q. Xie and G. S. Yan, Sci. China (B) 52, 1987-1990 (2009).

193. P. Zou, Q. G. Li, Y. Xue and G. S. Yan, Acta Chim. Sinica 67, 2427-2432 (2009).

194. Q. G. Li, Y. Xue, Y. Guo and G. S. Yan, Acta PhysChim. Sin. 26, 1965-1975 (2010).

195. Y. Zeng, Y. Xue and G. S. Yan, J. Phys. Chem. A 115, 4995-5004 (2011). 